\title{
Malignant degeneration in chronic ulceration of the leg and foot in leprosy patients; two case reports
}

\author{
J G ANDERSEN \\ All Africa Leprosy and Rehabilitation Training Centre (ALERT) \\ P.O. Box 165, Addis Ababa, Ethiopia
}

Received for publication 20 August 1981

\begin{abstract}
Summary The development of squamous cell carcinoma in long-established ulcers is described in two patients with leprosy, one on the lower leg in a patient with borderline-tuberculoid (BT) leprosy and the other on the sole of the foot in a patient with lepromatous (LL) leprosy. Although sometimes stated by clinicians to be rare, and infrequently reported in the literature, it is suggested that malignant change in chronic ulcers in leprosy may be found more often if patients are examined with care and the possibility kept in mind.
\end{abstract}

\section{Introduction}

Writing in 1964, Job and Riedel ${ }^{1}$ commented that carcinoma arising in plantar ulcers in leprosy was extremely uncommon; they were in fact unable to find reports in the literature at that time. They suggested, however, that more cases might be recorded if careful attention were paid to the examination of ulcers and the possibility of malignant change kept in mind, and two years later Riedel published an additional note on malignancy in plantar ulcers, describing four cases of squamous carcinoma arising during a period of two years in one centre in India. ${ }^{2}$ Although it is well known to surgeons and others that chronic ulceration occasionally gives rise to malignant change, it is therefore perhaps significant that a survey of the leprosy literature at the present time does not suggest that this is a common event in chronic ulcers of the leg and foot in leprosy. However, experience from this centre in previous years indicates that the complication is perhaps commoner than the literature suggests and we believe that if competent examiners look for this development, it will not be a rarity. We report here two cases in which malignant change occurred in ulcers on anaesthetic skin areas in patients with long-standing leprosy. 


\section{Case reports}

First patient: a middle-aged lady. Clinical diagnosis: lepromatous leprosy, negative on slit-skin smear examination, under treatment with DDS since 1960. She had for a number of years suffered from ulceration with smouldering osteomyelitis of both forefeet. Both plantar surfaces have been anaesthetic for several years.

In April 1978 the condition of the left foot was considered serious enough to urge her to accept a formal forefoot amputation, while on the right side it was considered feasible to perform a pretalar amputation, followed by tibiocalcaneal fusion with 45 degrees rotation of calcaneum. She refused operation.

In May 1978, the septic condition of the left foot had advanced to the degree where a formal below-knee amputation was considered necessary. The condition of the right foot appeared reasonably stable, and the previous advice was repeated. Once again she refused.

In November 1978 a firm clinical diagnosis of secondary carcinoma of the

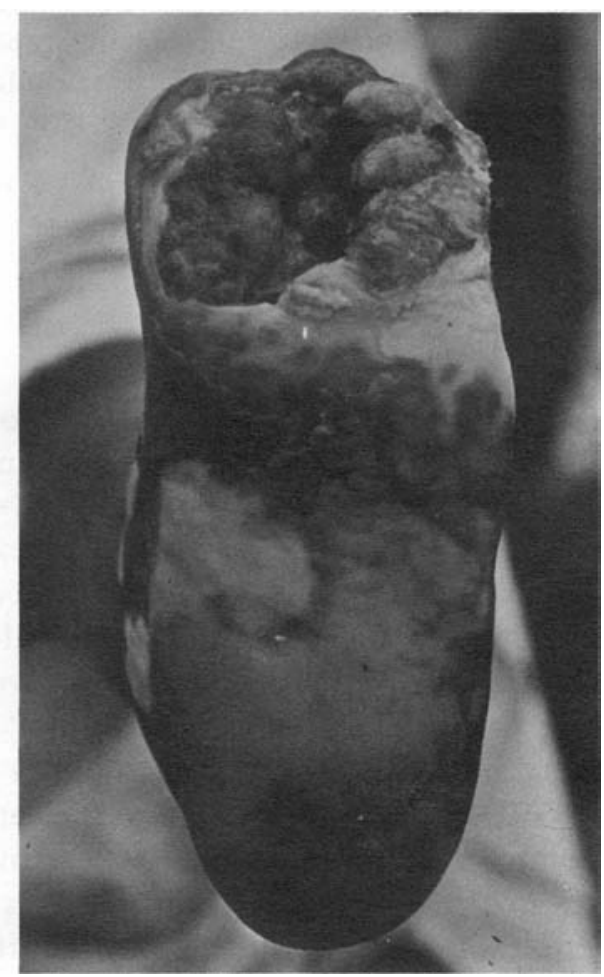

Figure 1. First patient. The ulcer as seen in November 1978, had increased in size and depth, with gross undermining. After removing pus and necrotic tissue red everted edges were revealed. 
left foot was made. When this was explained, she accepted below-knee amputation. The right foot was only slightly worse. It was considered possible to repeat the previous offer of a limited ablation of the fore- and mid-foot, but she still hesitated to accept this offer.

On first examination the ulceration of the left forefoot was deep, with exposed sequestra and necrotic tissue. The edges of the wound were sloping with no evidence of hyperkeratosis or hypertrophy, and with no significant undermining. X-ray examination demonstrated bone absorption and osteomyelitis with sequestration, but with no signs of malignancy.

On the second examination, the situation was much the same, but the ulceration had progressed and the radiological picture was worse. There was significant undermining of the edges, but still no signs of malignancy.

In November 1978 the picture was radically and dramatically different. The ulcer was very deep with gross undermining (Fig. 1). The whole area was covered with pus and necrotic tissue. When this had been cleared off, thick, raised, red everted edges were seen, a picture strongly suggestive of carcinoma. At no time were any significantly enlarged regional lymph nodes felt. Pathology report: squamous cell carcinoma.

Second patient: a middle-aged man. Clinical diagnosis: borderline-tuberculoid leprosy; slit-skin smears negative. First registered in 1965. He had for the last seven years suffered from repeated attacks of blister formation and eventual ulceration of the anterior surface of the lower leg on an area of extensive anaesthesia. He received repeated treatment with bland local applications. Five months ago he noticed that the ulcer increased in size and depth. Local and systemic antibiotics had no effect.

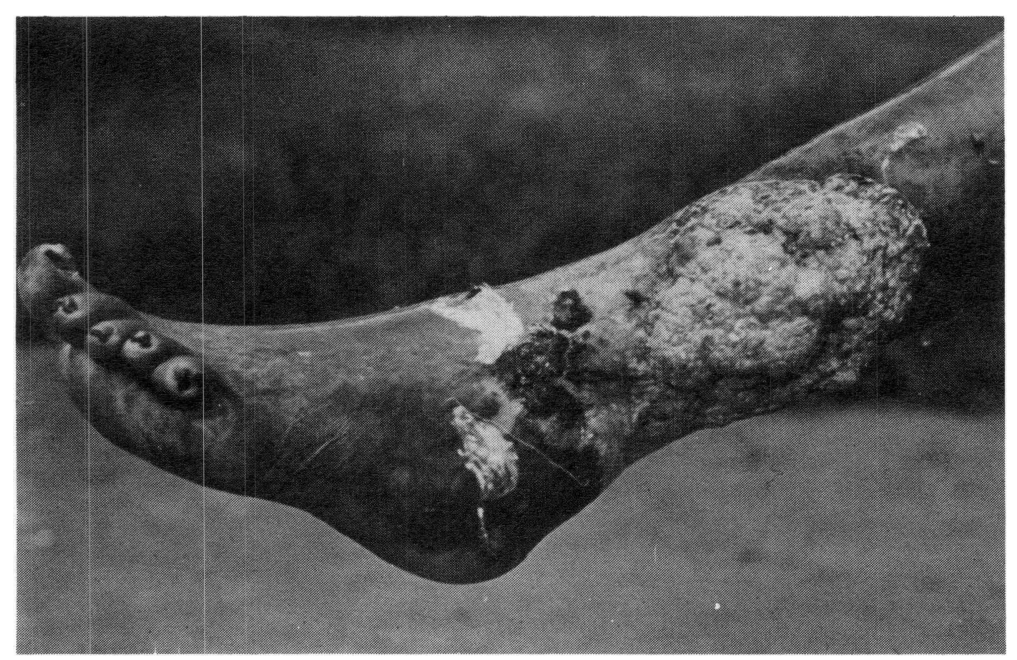

Figure 2. A large fungating mass on mid-shin, extending in all directions below skin surface. 
On examination the left lower leg was shiny with anaesthetic, atrophic skin. A large fungating mass was seen on mid-shin, apparently extending in all directions below the skin surface. The mass was adherent to deeper structures. No significant enlargement of regional lymph nodes was found. X-ray examination showed a hazy periosteal reaction of considerably larger extent than the actual ulcer.

A diagnosis of secondary carcinoma was made clinically. The patient accepted the offer of below-knee amputation. Pathology report: squamous cell carcinoma with involvement of periosteum.

\section{Discussion}

An increased incidence of malignant skin tumours in patients with lepromatous leprosy has been reported ${ }^{3}$ from a large series of patients in Brazil, but a recent review of the incidence of cancer of all types in patients with leprosy ${ }^{4}$ did not suggest a positive association. The second patient described here clearly had an ulcer on a site (lower leg) typical for the 'tropical' or 'phagedenic' ulcer, in which squamous cell carcinoma is said to occur in $9 \%$ of all cases. ${ }^{5}$ We have been unable to find any comparable figure for the likely incidence of malignant change in plantar ulcers in leprosy. In both cases described in this report, longstanding secondary infection appears to have been an important factor and there was certainly no evidence that specific infection with leprosy played any part. Resultant anaesthesia on the other hand may well have caused a diminished appreciation of pain in both patients, thus contributing to delay in their seeking treatment at an early stage. It may be of interest to recall that many tropical or phagedenic ulcers are extremely painful.

Ulcers undergoing malignant degeneration tend to produce a fungating mass with, in the initial stages, only radiologically non-specific periosteal reaction. At a later stage one may see an almost punched out lytic lesion in the tibia. Malignant degeneration in a plantar ulcer in leprosy is likely to remain undiagnosed for a long time, unless specifically looked for. The reason may be that the anaesthetic, ulcerated foot not only sequestrates the tissue before manifest malignancy becomes obvious, but also that the concomitant poor blood supply does not readily support the growth of malignant tissue. Self-cure, albeit with gross disability and tissue loss, is probably not infrequent. Carcinomata in chronic lower limb ulcers tend to metastasize slowly. Significantly enlarged regional lymph nodes should be left severely alone. It is impossible on clinical grounds to distinguish between metastases and septic enlargement of lymph nodes and attempts to excise them or even to take a biopsy, or to otherwise interfere with them is likely to produce a chronic, fistulating lesion. On the other hand, once the primary focus has been safely removed, septic lymph nodes will settle down and metastases may diminish in size, and even disappear in some cases. 
Prognosis for life is excellent in malignant degeneration of ulcers of the lower leg and sole of foot. The obvious treatment is surgical ablation. The actual level of amputation should be determined by the septic condition, the viability of tissue, and the suitability for prosthesis, rather than by the malignant process as such. Fortunately, the lowest convenient amputation often yields as good a result in respect of survival as higher levels of amputation.

\section{References}

1 Job CK, Riedel RG. Squamous cell carcinoma arising in plantar ulcers in leprosy. Int J Lepr, 1964; 32: 37-44.

2 Riedel RG. An additional note on malignancy in plantar ulcers in leprosy. Int $J$ Lepr, 1966;34: 287-8.

3 Michalany J. Malignant tumours of the skin among leprosy patients. Int J Lepr, 1966; 34 : 274-86.

4 Purtilo DT, Pangi C. Incidence of cancer in patients with leprosy. Cancer, 1975; 35: 1259-61.

5 Edginton GM, Gilles HM. Pathology in the Tropics. 2nd ed. Edward Arnold, 1976. 